\title{
Halitosis: Much beyond oral malodor
}

\section{Ongole $\mathbf{R}^{1}$, Shenoy $\mathbf{N}^{2}$}

${ }^{1}$ Associate Professor, ${ }^{2}$ Assistant Professor, Department of Oral Medicine \& Radiology, Manipal College of Dental Sciences, Mangalore

\begin{abstract}
Oral malodor one of the most common complaints with which patients approaches us thinking it can be detrimental to his self-image and confidence. Even though majority of oral malodor is of oral origin, there are multiple other systemic causes that have to be addressed while we diagnose and treat this condition. Most of these patients look up to oral care physicians for expert advice, it is critical for us to have the knowledge base and communication techniques to provide quality clinical assessment and implement effective intervention programs. This article reviews the various causes and the diagnostic modalities which will help us treat this multifaceted condition.
\end{abstract}

Key words: Halitosis, Oral malodor, Systemic diseases, Diagnosis

$\mathrm{T}^{\mathrm{s}}$ he word halitosis is derived from the Latin word halitus, which means exhalation. Halitosis is a term used to refer to offensive or bad breath. Fetor ex ore, fetor oris and stomatodysodia (dysodia in Greek refers to stench) are other terms that have been used in literature to describe halitosis. Halitosis is a general term used to describe an offensive odor emanating from the oral cavity. Approximately $90 \%$ of all bad breath originates from the mouth itself. Oral halitosis is the specific term used to describe halitosis that originates within the oral cavity ${ }^{1,2}$.

\section{Classification}

Halitosis can be broadly classified as true and false halitosis.

\section{Pseudo Halitosis}

Individuals complain of the existence of halitosis though it is not perceived by others. This condition can be managed effectively by counseling (using literature support, education and explanation of examination results) and simple oral hygiene measures.

\section{Halitophobia}

Some individuals continue to insist that they have halitosis even after they have been treated for genuine or pseudo-halitosis. Such individuals are categorized as halitophobic. Halitophobia may be considered when no physical or social evidence exists to suggest that halitosis is present ${ }^{3,4}$.
Classification of halitosis

\begin{tabular}{|l|}
\hline I. True Halitosis \\
\hline A. Physiologic (Transient or Temporary) \\
\hline Halitosis caused by dietary components \\
Halitosis caused by deleterious habits \\
Morning breath \\
Secondary to xerostomia caused by physiologic \\
factors \\
\hline B. Pathologic \\
Secondary to pathologic conditions or oral tissues \\
like gingival and periodontal diseases like Acute \\
Necrotizing Ulcerative Gingivitis \\
Residual post-operative blood \\
Debris under dental appliances \\
Ulcerative lesions of the oral cavity \\
Halitosis associated with coated tongue \\
Halitosis due to xerostomia secondary to salivary \\
gland diseases \\
Tonsilloliths \\
\hline II. False Halitosis or Halitophobia \\
\hline
\end{tabular}

Aetiopathogenesis

Oral halitosis is brought about by the action of bacteria on food debris and shed epithelial cells, which in turn releases volatile sulphur compounds. The commonly produced volatile sulphur compounds are hydrogen

\footnotetext{
Correspondence

Dr. Nandita Shenoy

Assistant Professor

Department of Oral Medicine \&Radiology

Manipal College of Dental Sciences

E-mail: nandita.shenoy@gmail.com
} 
sulphide $\left[\mathrm{H}_{2} \mathrm{~S}\right.$, rotten egg smell], dimethyl sulphide $\left[\left(\mathrm{CH}_{3}\right)_{2} \mathrm{~S}\right.$, rotten cabbage smell], and methyl mercaptan $\left[\mathrm{CH}_{3} \mathrm{SH}\right.$, fecal smell]. Methyl mercaptan is believed to be the most malodorous component.

Sulphur-containing amino acids [cysteine] are broken down by the anaerobic bacteria to release volatile sulphur compounds. Certain non- sulphur containing substances like diamines [cadaverine (cadaver smell) and putrescine (rotting meat smell)], acetone and acetaldehyde also contribute to halitosis emanating from the oral cavity. Other potentially odor producing substances include indole (used in small quantities in perfumes, however large quantities can produce an offensive odor), skatole (fecal odor), short-chain carboxylic acids such as butyric and valeric acids (sweaty feet odor) and ammonia. The activity of bacteria is at its peak at a $\mathrm{pH}$ of 7.2 and inhibited at a $\mathrm{pH}$ of $6.5^{5}$.

\section{Microflora associated with halitosis}

The principle bacteria that are implicated in the creation of oral malodor include Fusobacterium nucleatum, Prevotella intermedia and Tannerella forsythensis. Other bacteria that have been implicated in the production of volatile sulphur compounds include Prophyromonas gingivalis, Porphyromonas endodontalis, Treponema denticola, Aggregatibacter actinomycetemcomitans (earlierknownas Actinobacillus actinomycetemcomitans), Atopobium parvulum, Campylobacter rectus, Desulfovibrio species, Eikenella corrodens, Eubacterium sulci, Fusobacterium species and Peptostreptococcus micros.

Isolates of Klebsiella and Enterobacter are reported to have emitted foul odors in vitro which resembled bad breath in denture wearers. These gram-negative proteolytic anaerobes are located in the relatively stagnant areas of the mouth, such as periodontal pockets, posterior dorsal surface of the tongue, and interdental regions ${ }^{6}$.

Intra oral and systemic predisposing factors of halitosis

\section{Intra oral conditions}

Coating of the tongue is an important factor for oral malodor (80-90\%). Amir E et al (1999) and Poelmans J et al (2002) suggest that the individuals with history of oesophageal reflux disease and post nasal drip predispose to the build up of a substrate on the dorsal surface of the tongue ${ }^{5}$. The papillae of the tongue, crevices associated with mucous glands and lingual tonsils increase the accumulation of bacteria and exfoliated epithelial cells. Deposits on teeth and periodontal diseases like Acute Necrotising Ulcerative Gingivitis can also contribute to oral malodor ${ }^{7,8}$.

\section{Systemic conditions}

Respiratory tract diseases (lung abscesses, necrotizing pneumonia and carcinomas of the respiratory tract) can cause the breakdown of tissue leading to the production of volatile sulphur compounds. Other associated respiratory diseases like tonsillitis and postnasal drip caused by nasal infections, sinusitis or nasal polyps and produce oral halitosis.

Carcinomas of the upper respiratory tract, oral cavity and oropharynx, produce normal or branched organic acids, while lung carcinomas can produce acetone, methylethylketone, n-propanol, aniline and otoluidine?

Liver disease can produce a variety of aromatic compounds, such as $\mathrm{H}_{2} \mathrm{~S}$, aliphatic acids, $\mathrm{CH}_{3} \mathrm{SH}$, ethanethiol and $\left(\mathrm{CH}_{3}\right)_{2} \mathrm{~S}$. Trimethylaminuria is a rare, odor producing metabolic disease with symptoms of dysgeusia and dysosmia, which are due to excess production of trimethylamine, or $\left(\mathrm{CH}_{3}\right)_{3} \mathrm{~N}$. Hepatic cirrhosis will produce a characteristic musty or 'mousey' odor?.

Uremia that is caused by kidney failure also produces $\left(\mathrm{CH}_{3}\right)_{3} \mathrm{~N}$ along with dimethylamine. These individuals present with a uremic breath (ammoniacal odor) ${ }^{10,11}$.

Patients with uncontrolled diabetes mellitus (diabetic ketoacidoses) can emit ketonic breath (also described as sweet 'fruity' smell or rotten apple breath), which is caused by a metabolic disturbance leading to the production of acetones and other ketones.

Table 1: Summarizes the characteristic malodor associated with systemic diseases.

\begin{tabular}{|l|l|}
\hline Condition & Type of odor \\
\hline Diabetic Ketoacidoses & $\begin{array}{l}\text { Fruity odor, ketonic } \\
\text { breath, rotten apple odor }\end{array}$ \\
\hline $\begin{array}{l}\text { Bowel obstruction } \\
\text { associated with prolonged } \\
\text { vomiting or patients with } \\
\text { nasogastric tube }\end{array}$ & Fecal odor \\
\hline Chronic renal failure & $\begin{array}{l}\text { Ammonia like odor, urine } \\
\text { like odor, fishy odor }\end{array}$ \\
\hline Hepatic cirrhosis & Musty or mousey odor \\
\hline
\end{tabular}

Diagnosis of Halitosis

Oral malodor can be assessed using direct and indirect methods

\section{Direct methods}

a. Organoleptic method ${ }^{12}$ (whole-mouth breath test, spoon test, floss odor test, salivary odor test and self perception of odor) 
b. Gas chromatography

c. Sulphide monitors

d. "Electronic nose"

\section{Indirect methods}

a. Bacterial culture and smear ${ }^{14}$

b. Enzyme assay

\section{Organoleptic Method}

Organoleptic measurement can be carried out by sniffing the patient's breath and grading the level of halitosis. Though this technique is crude in nature, it is still the most reliable technique for assessing the level of oral halitosis. Assessment of oral halitosis should be carried out on two or three occasions for a reasonably accurate diagnosis.

\section{Pre-procedural requirements}

The patient is instructed to avoid taking antibiotics 3 weeks before procedure. They should also be instructed to refrain from ingesting garlic, onion and spicy foods for 48 hours before the assessment. Certain other requirements include avoiding use of perfumes, deodorants for 24 hours before the assessment and smoking and alcohol 12 hours before the procedure. They should also be discouraged from using breath fresheners and oral rinses 12 hours before assessment.

\section{Examiner}

The examiner conducting the test should have a normal sense of smell. He or she should avoid drinking coffee, tea or alcohol and abstain from smoking. Use of perfumes and scented cosmetics should be strictly avoided.

\section{Whole mouth breath test}

The patient and the examiner are seated on either side of a privacy screen. This screen will make the patient believe that he/she is undergoing a scientific test. A $50-70 \mathrm{cms}$ long, $2.5 \mathrm{cms}$ diameter transparent tube is inserted through the privacy screen.

The patient is asked to place one end of the tube into his mouth and exhale slowly as the examiner seated across the privacy screen will sniff the exhaled air on the other end and grade the halitosis.

The organoleptic evaluation of oral malodor also includes other simple tests such as tongue odor test, dental floss odor test and saliva odor test.

\section{Spoon test}

The spoon test is used to assess halitosis originating from the posterior part of the dorsum of the tongue ${ }^{15}$. A sterile plastic spoon is used to scrape the dorsum of the tongue. After about 5 seconds, the odor from the contents of the spoon is assessed, holding the spoon about $5 \mathrm{cms}$ away from the nose.

Organoleptic scoring scale (Miyazaki H et al.)

\begin{tabular}{|l|l|l|}
\hline Score 0 & Absence of odour & $\begin{array}{l}\text { Odour cannot be } \\
\text { detected }\end{array}$ \\
\hline Score 1 & Questionable odour & $\begin{array}{l}\text { Odour is detectable, } \\
\text { although the } \\
\text { examiner could } \\
\text { not recognize it as } \\
\text { malodour. }\end{array}$ \\
\hline Score 2 & Slight malodour & $\begin{array}{l}\text { Odour is deemed to } \\
\text { exceed the threshold } \\
\text { of malodour } \\
\text { recognition. }\end{array}$ \\
\hline Score 3 & Moderate malodour & $\begin{array}{l}\text { Malodour is } \\
\text { definitely detected }\end{array}$ \\
\hline Score 4 & Strong malodour & $\begin{array}{l}\text { Strong malodour } \\
\text { is detected, but } \\
\text { can be tolerated by } \\
\text { examiner }\end{array}$ \\
\hline Score 5 & Severe malodour & $\begin{array}{l}\text { Overwhelming } \\
\text { malodour is } \\
\text { detected and cannot } \\
\text { be tolerated by } \\
\text { examiner }\end{array}$ \\
\hline
\end{tabular}

Dental floss odor test

This test is used to assess the odor originating from the inter dental regions. The examiner passes a sufficient length of unwaxed floss through the inter dental regions of posterior teeth. The odor is assessed by holding the floss about $3 \mathrm{cms}$ from the nose.

\section{Saliva odor test}

The patient is instructed to expectorate about $1-2 \mathrm{ml}$ of saliva into a glass tube. The tube is covered immediately and incubated at $37 \mathrm{C}$ for five minutes. The glass tube is then held about $4 \mathrm{cms}$ away from the nose for assessing odor $^{14,16}$.

\section{Gas Chromatography}

Gas chromatography ${ }^{17}$ is a highly sensitive technique to assess breath malodor. Gas chromatography along with flame photometry is used to measure the most abundantly produced volatile sulphur compounds in the mouth $\left(\mathrm{CH}_{3} \mathrm{SH}, \mathrm{H}_{2} \mathrm{~S}\right.$, and $\left.\left(\mathrm{CH}_{3}\right)_{2} \mathrm{~S}\right)$. Other substances that are associated with oral malodor such as cadaverine, putrescine and skatole can also be detected.

The OralChroma ${ }^{\mathrm{TM}}$ portable gas chromatography device analyses individual concentrations of volatile sulphur compounds such as Hydrogen sulfide, Methyl mercaptan and Dimethyl sulfide and displays the concentrations on a display panel. 
The evaluation of halitosis can be performed in three basic steps using this unit.

Step 1: A plastic syringe that comes with the product is placed deep into the mouth and held with the lips to form a seal with the barrel of the syringe. The plunger is gently pulled and then pushed back. For the second time the plunger is pulled back before the syringe is taken out of the mouth.

Step 2: If the syringe is wet on the outer surface is wiped. The needle provided by the manufacturer is attached to the syringe and the plunger is pushed such that only 0.5 $\mathrm{cc}$ of the gaseous contents remain in the syringe.

Step 3: The remaining gaseous contents in the syringe are injected into an inlet on the main unit of the OralChroma $^{\mathrm{TM}}$.

\section{Advantages of Gas Chromatography}

1. Identifies individual components of the gas sample

2. The system can detect minute quantities of the gas even when the patient has used halitosis inhibiting agents.

\section{Disadvantages of Gas Chromatography}

1. The technique requires highly trained personnel.

2. Expensive equipment and the machine is not portable

\section{Sulphide Monitors}

Sulphide monitor ${ }^{18}$ is portable chair side equipment that can assess oral malodor, these monitors are cost effective and commercially marketed as Halimeter ${ }^{R}$ (Interscan, Chatsworth, California.).

The monitor is equipped with an electrochemical sensor. The patient is asked to exhale into a transparent tube that carries the breath to a suction pump which in turn carries the air to the monitor. These monitors analyze the total sulphur content of the individuals breath but cannot differentiate between various sulfides. The instrument measures parts per billion levels of hydrogen sulfide and, to a lesser extent, methyl mercaptan.

This monitor may show erroneous results in the presence of high ethanol or essential oil levels in the breath. The monitor needs periodic recalibration in order to maintain its sensitivity.

\section{Electronic nose}

Electronic noses ${ }^{19}$ are chemical sensors that have been in the recent times for a quantitative assessment of malodor associated with food and beverages. Mantini et al described various biomedical uses of these chemical sensors. Tanaka $\mathrm{M}$ et al used these electronic noses to clinically assess oral malodor and examined the association between oral malodor strength and oral health status.

The FF-1 odor discrimination analyzer (electronic nose, Shimadzu Corporation) was used by Tanaka $\mathrm{M}$ et al. The set up comprised of a pre-concentrator, an array of 6 metal oxide semiconductor sensors selected for their different sensitivities and selectivity's to fragrant substances, and a pattern recognition software. The instrument can be set to various modes such as the "allnote measurement mode" which is the standard setting used for measuring all volatile substances and the "topnote measurement mode" which primarily measures volatile substances with a low boiling point. The results of their preliminary study showed that main compounds related to oral malodor were volatile substances with a low boiling point.

\section{Indirect methods to assess oral halitosis}

Bacterial culture, smears and enzyme assays are indirect methods of assessing oral halitosis. These methods will help in the identification of organisms that produce oral malodor. One such technique is BANA test.

BANA (N-benzoyl-DL-arginine naphthylamide) test BANA $^{20}$ test is a chair side investigation that assesses the proteolytic activity of anaerobic bacteria. It is a rapid chair side test for evaluation of non-sulfurous malodorous compounds.

Test

To detect malodor, the tongue or inter dental regions are wiped with a cotton swab. The sample is placed on the BANA test strip, which is then inserted into a slot on a small toaster-sized incubator. The incubator automatically heats the sample to $55^{\circ}$ for 5 minutes. If P. gingivalis, B. forsythus or T. denticola are present, the test strip turns blue. The bluer it turns, the higher the concentration and the greater the number of organisms. A color guide is printed on the container. It can also be used to evaluate the prognosis of the condition. Individuals who have been treated successfully for oral halitosis will reveal a BANA test that converts from a positive to a negative.

The BANA Test is a modification of the BANA hydrolysis test developed by Dr. Walter Loesche and colleagues at the Univ. of Michigan School of Dentistry. It exploits an unusual enzyme found in Treponema denticola, Porphyromonas gingivalis and Bacteroides forsythus, three anaerobic bacteria highly associated with adult periodontitis. These three bacteria possess an enzyme capable of hydrolyzing the synthetic peptide benzoyl-DL-arginine-naphthylamide (BANA) present 
on BANA test strips. If any of the three species is present, they hydrolize the BANA enzyme producing B-naphthylamide which in turn reacts with imbedded diazo dye to produce a permanent blue color indicating a positive test.

\section{Management of Oral Malodor}

The first step towards effectively managing oral halitosis is to determine the cause for halitosis (oral or systemic) and the nature of halitosis. A good medical, dental and diet history will help in determining the origin for halitosis in $^{21}$.

\section{General measures}

1. Patients should be advised to drink plenty of water and rinse mouth thoroughly after every meal.

2. Patients should be encouraged to clean the dorsum of the tongue gently with a soft bristled tooth brush.

3. Patients should be encouraged to undergo periodic scaling procedure.

4. Proper brushing and flossing technique should be advised.

5. Patient can be encouraged to include fibrous vegetables in the diet.

\section{Specific measures}

\section{Elimination of foci of infection}

Oral prophylactic procedures such as supra and sub gingival scaling and elimination of periodontal pockets should be undertaken ${ }^{23,24}$. Carious teeth have to be restored ${ }^{16}$. Teeth with periapical pathology should be endodontically treated. Abscesses of acute nature should be managed using appropriate antibiotics.

\section{Antiseptic mouth rinses ${ }^{25}$}

Chlorhexidine gluconate mouth wash $(0.2 \%)$ which is an effective anti plaque agent is used to manage oral malodor.

Triclosan (2, 4, 4-trichloro-2-hydroxydiphenylether) is a broad spectrum nonionic antimicrobial agent. Literature review reveals that triclosan effectively minimizes oral malodor. A more effective mouth rinse against oral malodor is obtained when triclosan is used in combination with zinc.

Mouth rinses containing alcohol are best avoided ${ }^{26,27}$. Alcohol containing rinses will dry up the oral mucosa, thereby worsening the oral halitosis

\section{Zinc Rinses ${ }^{28}$}

Zinc rinses (in chloride, citrate or acetate form) have been found to reduce concentration of volatile sulphur compounds. Zinc rinses are believed to inhibit the reduction of disulfide group to thiols. An independent study showed that zinc containing chewing gum reduced oral malodor significantly.

\section{Miscellaneous products ${ }^{29,30}$}

Commercially available mints and breath freshners containing menthol have also been reported to reduce oral malodor, mainly by a masking effect. Spices such as Cardamom and cloves have been used since time immemorial to mask bad odor.

\section{Recent innovations in the management of halitosis}

\section{Anti Halitosis Mouth rinse}

The first active ingredient of AHM is highly oxidizing sodium chlorite ( $600 \mathrm{ppm}$ of chlorite ion) which oxidizes the sulfides of the VSCs to non-odorous sulfates and raises the oxidation/reduction ratio of the saliva toward the more oxidizing state. This also suppresses the overgrowth of the anaerobic bacteria on the tongue. The other active ingredient zinc acetate (300 ppm of $\mathrm{zn}$ ion) oxidizes the VSCs and creates a more oxygen rich oral environment, but also interferes with the proteolytic activities of the anaerobic bacteria. This combination provides a synergestic anti-halitosis effect for more than 6 hours. This material is still under clinical trials and not many definite studies are published so far. ${ }^{31}$

\section{Chlorine dioxide mouth rinse}

Chlorine dioxide as a mouth rinse neutralizes volatile sulfur compounds in mouth air. The efficacy of a chlorine dioxide-containing mouthrinse in the reduction of oral malodor has been evaluated in randomized, controlled, double-blind trials. One study demonstrates that a onetime use of a chlorine dioxide-containing mouthrinse significantly improves mouth odor pleasantness and reduces mouth odor intensity for at least 4 hours $^{32}$.

Cochrane review ${ }^{33}$ on the various mouthrinses available

Cochrane review states, mouth rinses containing antibacterial agents such as chlorhexidine and cetylpyridinium chloride may play an important role in reducing the levels of halitosis-producing bacteria on the tongue, and chlorine dioxide and zinc containing mouth rinses can be effective in neutralisation of odouriferous sulphur compounds. Well designed randomised controlled trials with a larger sample size, a longer intervention and follow-up period are still needed.

\section{Management of halitophobic individuals}

These individuals can be reassured by using a simple 'air bag' technique, which is a self assessment organoleptic technique ${ }^{32}$. In this technique a food grade thin transparent plastic cover of $8 \times 10$ inches size is taken. The halitophobic individual is instructed to seal his/her mouth with the open end of plastic bag. He/She 
should inhale air through the nose and exhale through the mouth in short bursts. The procedure is continued till the plastic bag is fully inflated. The mouth of this plastic air filled bag is then held tight with finger pressure such that no air escapes out of the bag. Next, patient is seated comfortably in a well-ventilated odor free room. Air from the plastic bag should be squeezed out in front of the patient's nose while he/she inhales slowly. As the air is odor free, patient will get convinced that he/she is not suffering from halitosis. To further strengthen the belief, odor free air samples can be collected from healthy (to prevent transmission of air borne diseases) volunteers/relatives and friends of the patient and having the patient blindly rate the odor quality of each sample, including his/her own.

\section{Conclusion}

This article highlights on the possible causes and the various management modalities of halitosis patients. This aspect is very useful for general practitioners, especially with regard to patients with pseudohalitosis, who may seek treatment from them. Evaluation of the psychological condition of patients with halitosis is important and needs multidisciplinary approach.

\section{References}

1. ADA Council on Scientific Affairs. Oral malodor. J Am Dent Assoc. 2003; 134(2):20914.

2. Amir E, Shimonov R, Rosenberg M. Halitosis in children. The Journal of Pediatrics 1999; 134:338-43.

3. Bernie KM. The causes and management of oral malodor. Dent Today 2002; 21(2):92-7.

4. Almas K, Al-Hawish A, Al-Khamis W. Oral hygiene practices, smoking habit, and selfperceived oral malodor among dental students. J Contemp Dent Pract. 2003; 4(4):77-90.

5. Bosy A, Kulkarni GV, Rosenberg M, et. al. Relationship of oral malodor to periodontitis: evidence of independence in discrete subpopulations. J Periodontol. 1994; 65(1):3746.

6. De Boever EH, Loesche WJ. Assessing the contribution of anaerobic microflora of the tongue to oral malodor. J Am Dent Assoc. 1995; 126(10):1384-93.

7. Christensen GJ. Why clean your tongue? J Am Dent Assoc. 1998 ;129(11):1605-7

8. Eli I, Baht R, Koriat H, Rosenberg M. Self-perception of breath odor J Am Dent Assoc. 2001; 132 (5):621-6.

9. Feller L, Blignaut E. Halitosis: a review. SADJ. 2005 Feb; 60(1):17-9.
10. Greenman J, El-Maaytah M, Duffield J, Spencer P, Rosenberg M, Corry D, Saad S, Lenton P, Majerus G, Nachnani S. Assessing the relationship between concentrations of malodor compounds and odor scores from judges. J Am Dent Assoc. 2005; 136(6):749-57.

11. Goldberg S, Cardash H, Browning H, et. al. Isolation of Enterobacteriaceae from the mouth and potential association with malodor. J Dent Res. 1997; 76(11):1770- 75.

12. Greenman J, Duffield J, Spencer P, Rosenberg M, Corry D, Saad S, Lenton P, Majerus G, Nachnani S, El-Maaytah M. Study on the Organoleptic Intensity Scale for Measuring Oral Malodor. J Dent Res 2004; 83(1): 81-85.

13. Kita J, Aoyama Y, Kinoshita M, Nakano H, Akamaru H (2000). Quantification of the MOS sensor based electronic nose utilizing trap tube. In: Technical digest of the 17th Sensor Symposium, May 30, 2000. Tokyo: The Institute of Electrical Engineers of Japan, pp. 301-305.

14. Kleinberg I, Westbay G. Salivary and metabolic factors involved in oral malodor formation. J Periodontol. 1992; 63(9):768-75.

15. Hartley G, El-Maaytah, M, Greenman J. Tongue microflora of subjects with low and high malodour levels. J Dent Res. 1995 74:587.

16. Lee PPC, Mak WY, Newsome P. The aetiology and treatment of oral halitosis: an update. Hong Kong Med J 2004; 10:414-8.

17. Oho T, Yoshida Y, Shimazaki Y, Yamashita Y, Koga T. Characteristics of patients complaining of halitosis and the usefulness of gas chromatography for diagnosing halitosis. Oral Surg Oral Med Oral Pathol Oral Radiol Endod 2001; 91:531-34.

18. Rosenberg M, Septon I, Eli I, Bar-Ness R, Gelernter I, Brenner S, et al. Halitosis measurement by an industrial sulphide monitor. J Periodontol (1991a); 62:487-89.

19. Mantini A, Di Natale C, Macagnano A, Paolesse R, Finazzi-Agro A, D'Amoco A (2000). Biomedical application of an electronic nose. Crit Rev Biomed Eng 28:481-485.

20. Kozlovsky A, Gordon D, Gelernter I, Loesche WJ, Rosenberg M. Correlation between the BANA test and oral malodor parameters. J Dent Res 1994; 73:1036-42.

21. Lenton P, Majerus G, Bakdash B. Counseling and Treating Bad Breath Patients: A Step-ByStep Approach. J Contemp Dent Pract 2001; 2(2): 46-61.

22. Klokkevold PR. Oral malodor: a periodontal perspective. J Calif Dent Assoc. 1997; 25(2):153-9. 
23. Miyazaki H, Arao M, Okamura K, Kawaguchi Y, Toyofuku A, Hoshi K, Yaegaki K. Tentative classification of halitosis and its treatment needs. Niigata Dent J 1999; 32:7-11. Japanese.

24. Porter SR, Scully C. Oral malodour (halitosis). BMJ 2006; 333(7569): 632 - 35.

25. Ratcliff PA, Johnson PW. The relationship between oral malodor, gingivitis, and periodontitis. A review. J Periodontol. 1999; 70(5):485-9.

26. Loesche WJ. The effects of antimicrobial mouthrinses on oral malodor and their status relative to U.S. Food and Drug Administration regulations. Quintessence Int 1999; 30:311-8.

27. Neiders M, Ramos B. Operation of bad breath clinics. Quintessence Int 1999; 30:295-301.

28. Scully C, Rosenberg M. Halitosis. Dent Update. 2003; 30(4):205-10.

29. Scully C, Porter R, Greenman J. What to do about halitosis? Brit Med J 1994; 308: 217-18.
30. Tonzetich J, Ng SK. Reduction of malodor by oral cleansing procedures. Oral Surg Oral Med Oral Pathol. 1976; 42(2):172-81.

31. Kleinberg I, Codipilly M: Modeling of the oral malodor system and method Of analysis. Quintessence International; 30 (5): 357-369, 1999.

32. Frascella J, Gilbert R, Fernandez P, Odor reduction potential of a chlorine dioxide mouthrinse. J Clin Dent. 1998; 9(2):39-42.

33. Fedorowicz Z, Aljufairi H, Nasser M, Outhouse TL, Pedrazzi V, Mouthrinses for the treatment of halitosis;Cochrane Database Syst Rev. 2008 Oct 8;(4):CD006701.

34. Suhas S, Sudarshan S, Pai KM. "Air bag" organoleptic behavioral experiment for managing fear of oral malodor. J Behav Ther Exp Psychiatry. 2004; 35 (1):13-5. 